\title{
FILTER MAGNETOGRAPH MEASUREMENTS AT MEUDON OBSERVATORY
}

Audouin DOLLFUS et Jacques MOITY

Observatoire de Paris, 92195 Meudon, France

\section{Summary.}

We report observational studies of solar active regions $n^{\circ} 6150$ and $n^{\circ} 6850$ during cycle 22 . Observations were carried out with a tunable monochromatic birefringent filter coupled with a line-shifter and a Stokesmeter as well as with a spectro-magnetograph, both at Meudon Observatory. AR $n^{\circ} 6150$ is typical of emerging flux regions, while AR $n^{\circ}$ 6850 , with a complex preceding -spot, exhibits characteristic configurations of the transverse magnetic field for flaring activity : shear along the neutral line, and curvature coupled with anomalous Evershed mass motions.

\section{The instrument.}

The tunable monochromatic birefringent filter FPSS (Filtre Polarisant Solaire Sélectif) of Meudon Observatory, prototype of the Universal Birefringent Filters, was combined with a line-shifter and a Stokesmeter, in order to analyze by imagery the magnetic fields and mass motions in the photosphere (Dollfus, 1984; Dollfus et al., 1985). The instrument was used at Meudon with a $29 \mathrm{~cm}$ polarization free solar refractor. An analysis of the photospheric $\mathrm{p}$ mode oscillations was first conducted by Doppler imaging and movies (Dollfus, 1990-a).

Since 1987, active regions were analyzed, in a joint program with the Meudon spectro-magnetograph and spectroheliograph and the solar flare patrol at Meudon and Haute-Provence Observatories.

Active regions are typically documented by a set of images including : the photospheric and facular images, the $H_{\alpha}$ configuration, the line of sight velocities, the longitudinal $\left(B_{\prime \prime}\right)$ and transverse $\left(\overrightarrow{B_{1}}\right)$ magnetic fields, the modulus of the longitudinal field gradient $\mid$ gra d $B, l$, the magnetic neutral line, plus location of flare activities. The two following observations illustrate typical flaring configurations.

A flaring emerging flux region :AR $n^{\circ}$ 6150. On July 10, 1990, AR 6150 appeared as a pure single spot of negative magnetization (preceding spot polarity). On July 11 at 08:25, a cluster of small spots or macro-pores emerged at left (top left image in Fig.1). Then, after seven hours, the assemblage produced a multi-component following spot (image at 15:00). The magnetic field, initially faint and widely spread (magnetogram at top right), grows at an average rate of $1.3 \times 10^{2} 0 \mathrm{Mxh}-1$ to reach 700 gauss and a total flux of $+8 \times 10^{2} 0 \mathrm{Mx}$ (magnetogram at 15:00).

This emerging flux region (EFR) trigged a strong flaring activity. The $H_{\alpha}$ brightening rose significantly after 05:40. An $H_{\alpha}$ and $X$ ray flare of class $C 2.9$ occurred at $06: 30$ at the EFR site. Then the $H_{\alpha}$ brightening remained steady for several hours, with sub-flare activities. After 13:50, it splitted into two bright areas over the preceding and following spots respectively. 
Then, the group reached the stage of a steady bipolar center with the formation of an $H_{\alpha}$ arch filaments system (AFS). The next day on July 12 , Fig. 2, the configuration stayed rather unchanged, with same magnetic field, the new-born following spot slightly enhanced and an insignificant sub-flare activity. On July 13 , the following spots compacted into a single larger spot. The symmetrical group was totally deprived of any noticed flaring activity.
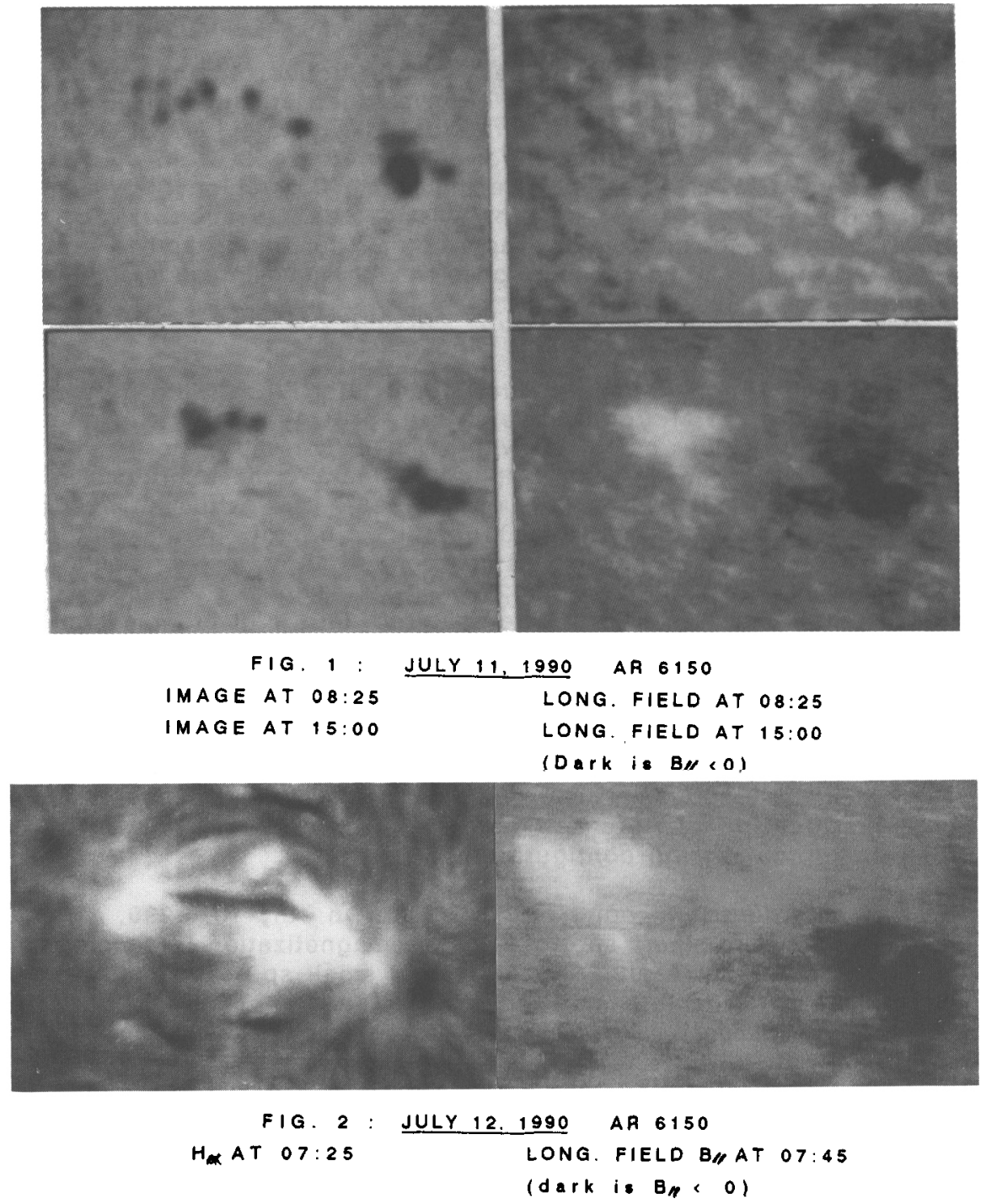
A sub-flaring active delta spot: AR $n^{\circ} 6850$. Fig. 3 is obtained on Oct. 3 . 1991 at 11:15. The delta spot includes 8 umbrae in the same penumbra and the magnetic field (image at top right) shows mixed polarities. There is a band of negative polarity (dark) splitting the positive field into two parts. This negative field reaches -2000 gauss at some places and the positive region does not exceed +2500 gauss. Such a configuration could be the result of an interaction between two bipolar groups, in which the preceding spot of a group moved from left towards the group at right and squeezed its components. This configuration could also result from the emergence of a maanetic structure which was already mixed and twined below the surface.

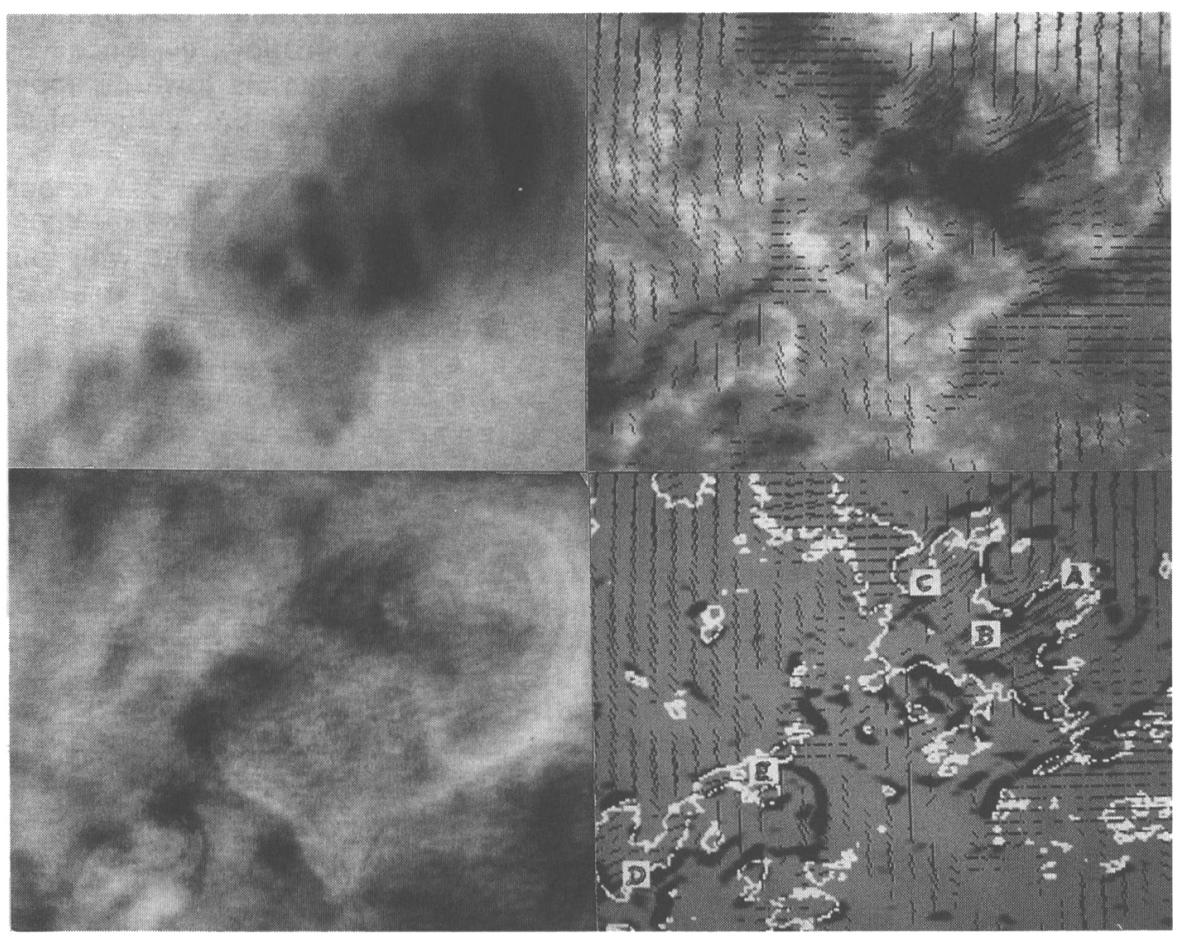

FIG. $3:$ OCT. 3.1991 AT 11:15 AR 6850

I MAGE

LONG VELOCITY V//

(dark is towards us)
LONG. B" AND TRANSV. $\vec{B}_{2}$ FIELDS

(dark is $B / y<0$ )

SUPERPOSITION OF $\overrightarrow{B_{I}}$. NEUTRAL

LINE $B / r=0$ (White). STRONG

GRAD Br(dark) AND LOCATION

OF FLARES. 
A strong transverse field connects two polarities of the spot. Magnetic arches are suggested. There were repetitive and often simultaneous microflares in this area. Their preferential locations appear in the figure at bottom right in which they are superimposed upon the images of the transverse field and of the horizontal gradient $|\mathrm{grad} B / /|$.

Recurrent sub-flares arose in $A$ close to the feet of the magnetic arches where 1 grăd $B_{\prime \prime} \mid$ is steep (dark lane) with 0.25 G.km- 1. Recurrent often simultaneous sub-flares occurred also in $B$, a place of steep gradient, but between the two feet of the arch. They extended several times along an $H_{\alpha}$ ribbon along the path $B-C$.

North of $C$ there is a curvature in the transverse field. This bending was possibly related to a discontinuity in the Evershed flow, evidenced by the Doppler image (dark is towards observer; sun center is towards upper left). As the relationship curr $\vec{B}=\vec{j}$ applies, there is production of an electric current. There were only repetitive small events in $C$.

The major flaring event which happened on October 3 was in D. A ribbon connected $D$ to the following spot (out of the frame), from 08:49 to 09:03. and again from 10:04 to $10: 20$ and from $14: 28$ to $14: 48$, on a site with four characteristic configurations : - on a neutral line - in an area of significant horizontal $B / /$ gradient (dark lane) - where transverse field is almost parallel to the neutral line (white line) and with horizontal Evershed mass motion perpendicular to the transverse field and to the neutral line. These properties characterize a local squeeze at the photospheric level, with a shear of magnetic fluxes of opposite polarities on both sides of the neutral line. There is also a recurrent sub-flaring area in $E$ which, to a lesser extent, can be characterized the same way.

To conclude, this large and complex multi-spot delta group AR 6850 was deprived of large flare events, but almost continuously animated by multiple micro and small flaring activities. Locations of the recurrent bright points or lanes were preferentially at the feet of magnetic loop systems, on neutral lines where steep field gradients occur, and along horizontal shearing at both sides of the neutral line. Evershed mass motion forces are indicated to be involved in the process (see also Dollfus, 1990-b).

\section{References}

- Dollfus A., 1984. Stokes parameters modulor for birefringent filters. In "Measurements of solar vector magnetic fields". NASA Conf. Pub . 2374.

- Dollfus A., Colson F., Crussaire D. and Launay F., 1985.

A monochromator for solar quantitative imagery: The instrument FPSS Astron. Astrophys. 151, 235-253.

- Dollfus A., Crussaire D., Pernot E. and Lioure A., 1986. Cartographie détaillée de paramètres physiques de la photosphère par imagerie monochromatique. Compto-Rendus Acad. Sci Paris, 303, II, 153-158.

- Dollfus A., 1990-a. High resolution analysis of solar photospheric oscillations. Solar Physics, 129, 1-30.

- Dollfus A., 1990-b. Small scale magnetic structures in active centers. In "Solar Photosphere : Structure, convection and magnetic field". J.O. Stenflo Editor, IAU. 